\title{
Activity Phenomena Observed at Radio Frequencies in Sprial Galaxies
}

\author{
V.H.Malumian \\ Byurakan Astrophysical Observatory, Byurakan, 378433, Armenia
}

\begin{abstract}
It has been shown that the stage of activity of spiral galaxies depends on both the distance between them and their nearest neighbours and the structure of their central parts.
\end{abstract}

In the end of the seventies on the basis of radio observations of many spiral galaxies it has been shown, that among galaxies which are the members of pairs of galaxies, especially of close and interacting pairs, radio sources with the level of radio emission above a fixed limit occur significantly more frequently than among single ones (Stocke 1978). Later on it has also been shown that spiral members of pairs of galaxies are 2 to 2.5 times stronger radio sources than single galaxies of the same absolute magnitude (Altschuler \& Pantoja 1984; Malumian 1986, 1987a, 1989a, 1990). Interesting results were obtained on the basis of statistical investigations of the radio properties of spiral galaxies which are the members of loose groups of galaxies from the lists of Turner and Gott (Turner \& Gott 1976) and Geller and Huchra (Geller \& Huchra 1976). It turned out that if the members of groups were considered altogether irrespective of ranks among them the radio emission with the flux density above a fixed level occurs significantly more frequently than among single galaxies but noticably rarer than among the members of pairs of galaxies of the same absolute magnitude (Tovmassian \& Shakhbazian 1981; Malumian 1987b). When the spiral members of the groups were considered separately by rank, it turned out that radio emission among the first ranked spiral members of groups was observed to be not rarer than among the members of pairs of galaxies (Malumian 1987b).The relative numbers of radio sources is the highest among the first ranked spiral galaxies. The detailed analysis of radio properties of isolated spiral galaxies and spiral members of pairs and groups indicates that radio luminosities of spiral galaxies are determined by the presence of close neighbours rather than by the space density of galaxies around them. The rate of occurence of the radio sources and their radio luminosities in all probability do not depend on whether are members of pairs or groups of galaxies or they are isolated but depend on the presence of the neighbour. The probability of radio emission for spiral galaxies, if other conditions are the same, depends on the separation between them and their nearest neighbours. The shorter this separation the higher the probability of radio emission (Malumian, 1996). Now it is clear why the spiral members of groups from the list of Geller and Huchra (1976) are the less powerful radio sources than the members of pairs from Karachentsev's catalogue ( Karachentsev's catalogue 1987). The matter is that in the samples considered the separations between the members of the vast majority of pairs are much less than that between the members of groups and their nearest neighbours. For example, the number of 
pairs with projected separation between their members $R<50 k p c$ is nearly $80 \%$ but among galaxies within groups the objects with projected separation between them and their nearest neighbours $R<50 \mathrm{kpc}$ are only 16\%. In Malumian (1996) it was shown that members of groups with $R<200 \mathrm{kpc}$ are on average 1.5 times stronger radio sources than members with $R>200 k p c$. The relation between radio luminosity and the structure of the central parts of spiral galaxies was ascertained. The structure of the central parts of galaxies is determined by the Byurakan classification. It was shown that galaxies with stellar, semistell ar and split nuclei or with central condensations (marked 5, 4, 2s and 2 according to Byurakan classification) are 2 to 3 times more powerful radio emitters than galaxies without such signs (marked 3 and 1 according to Byurakan classification) (Malumian 1989). It was also ascertained that among galaxies of classes $2,2 \mathrm{~s}, 4$ and 5 are dominant objects with flat spectra of radio emission, and vice versa among galaxies of classes 1 and 3 are dominant the objects having steep spectra of radio emission (Malumian 1983). From our investigations of spectra of radio emission of spiral galaxies it follows that among galaxies of the classes 2, 2s, 4 and 5 the compact, recently formed radio sources occur more frequently. They have flat spectra of radio emission which became steeper for a comparatively short time because of losses of energy mainly due to synchrotron emission by emitting relativistic electrons. On the contrary, among the galaxies of classes 1 and 3 there is a large excess of objects which have steep spectra of radio emission. Summarizing the aforementioned facts one can state that the stage of activity of spiral galaxies depends on both the distance between them and their nearest neighbours and the structure of their central regions. The shorter this separation the higher the stage of activity. The stage of activity is also higher in spiral galaxies with any pecularities in their central parts.

\section{References}

Altschuler, D.R., \& Pantoja, C.A. 1984, AJ, 89, 1531

Geller, M. \& Huchra, J. 1976, ApJS, 32, 409

Karachentsev, I.D. 1987, Dvojnie Galaktiki (Double Galaxies): Moscow

Malumian, V.H. 1983, Astrofizika, 19, 251

Malumian, V.H. 1986, Astrofizika, 25, 19

Malumian, V.H. 1987a, IAU Symposium 121, p.24

Malumian, V.H. 1987b, Astrofizika, 26, 311

Malumian, V.H. 1989a, Astrofizika, 30, 223

Malumian, V.H. 1989b, Astrofizika, 31, 241

Malumian, V.H. 1990, Astrofizika, 32, 507

Malumian, V.H. 1996, Astron. Nachr., 317, 101

Stocke, J.T. 1978, AJ, 83, 348

Turner, E., \& Gott, J.R. 1976, ApJS, 32, 409

Tovmassian, H.M., \& Shakhbazian, E. 1981, Astrofizika, 17, 265 